Algebraic $8 \mathcal{G}$ Geometric $\mathcal{T}$ opology

Volume 1 (2001) 435-444

Published: 22 August 2001

ATG

\title{
On periodic homeomorphisms of spheres
}

\author{
L. Montejano \\ E.V. SHCHEPIN
}

\begin{abstract}
The purpose of this paper is to study how small orbits of periodic homemorphisms of spheres can be.
\end{abstract}

AMS Classification $57 \mathrm{~S} 25 ; 57 \mathrm{~N} 15$

Keywords Periodic homeorphism, sphere

\section{Introduction}

A well known theorem of Newman [4],[3] states that periodic homeomorphisms of manifolds cannot have all orbits arbitrarily small. The purpose of this paper is to make this theorem precise for the case of spheres by exploring how small orbits of periodic homeomorphisms of spheres can be.

Throughout this paper, we will denote by $S^{n}$ the unit sphere of euclidean $(n+1)$-dimensional space $R^{n+1}$. Let $h: S^{n} \rightarrow S^{n}$ be a homeomorphism, we will denote by $h^{i}: S^{n} \rightarrow S^{n}$ the $i$-th iteration of $h$ and we will suppose that $h^{0}=i d$. A homeomorphism $h: S^{n} \rightarrow S^{n}$ is called periodic if $h^{n}=i d$ for some integer $n>1$. The minimal integer $n>1$ for which $h^{n}=i d$ is called the period of $h$. For every $x \in S^{n}$, the set $\left\{h^{i}(x)\right\}_{i>0}$ is called the orbit of $x$ and is denoted by $h^{*}(x)$. The shift, $\|h\|$, of a homeomorphism $h: S^{n} \rightarrow S^{n}$, is defined as its distance from the identity, that is, $\|h\|=\operatorname{Sup}\|h(x)-x\|$, and the orbital diameter, $\Theta(h)$, of $h$ is defined as the maximal diameter of its orbits. We will be mainly interested in lower estimations of these two metric characteristics of periodic homeomorphisms.

Let us denote by $\rho_{n}$ the length of the side of a planar regular $n$-gon inscribed in the unit circle $S^{1}$, and by $d_{n}$ its diameter. Finally, let us denote by $t_{n}$ the length of the edge of a regular $(n+1)$-simplex inscribed in $S^{n}$.

Our main results are the following:

(C) Geometry ${ }^{6}$ Topology $\mathcal{P}$ ublications 
Theorem 1.1 For any periodic homeomorphism $h: S^{n} \rightarrow S^{n}$ of prime period $p$,

$$
\|h\| \geq \rho_{p}
$$

Furthermore, if $\|h\|=\rho_{p}$, then there exists a point $x \in S^{n}$, such that the orbit $h^{*}(x)$ consists of the vertices of a planar regular convex $p$-gon.

The next theorem gives a bound for the orbital diameter of a periodic homeomorphism in terms of the dimension of the $n$-sphere.

Theorem 1.2 For any periodic homeomorphism $h: S^{n} \rightarrow S^{n}$,

$$
\Theta(h) \geq t_{n} .
$$

Furthermore, if $n \neq 1,3,7$,

$$
\Theta(h) \geq t_{n-1}
$$

It is well known, and in fact it is easy to see, that any homeomorphism of period two has an orbit consisting of a pair of antipodal points. This implies that the orbital diameter of a homeomorphism of period two coincides with the diameter of the sphere. In this direction we have the following results.

Theorem 1.3 For any periodic homeomorphism $h: S^{n} \rightarrow S^{n}$, of period 3,

$$
\Theta(h) \geq d_{3}=\sqrt{3} .
$$

Theorem 1.4 For any periodic homeomorphism $h: S^{1} \rightarrow S^{1}$ of period $p$,

$$
\Theta(h) \geq d_{p}
$$

Our conjecture is that the orbital diameter of a homeomorphism $h: S^{n} \rightarrow S^{n}$, of prime period $p$, is at least $d_{p}$. We shall show that the conjecture is true for $p=2,3$, for $n=1$ and for isometries.

\section{Some preliminary results}

Let $h: S^{n} \rightarrow S^{n}$ be a homeomorphism of period $p$. An orbit $h^{*}(x)$ is balanced if its baricentre coincides with the centre of the sphere, that is, if

$$
\sum_{1}^{p} h^{i}(x)=0 .
$$

Algebraic 83 Geometric Topology, Volume 1 (2001) 
For every periodic homeomorphism $h$ without balanced orbits we define its baricentric mapping $\beta: S^{n} \rightarrow S^{n}$ as:

$$
\beta(x)=\frac{\sum_{1}^{p} h^{i}(x)}{\left\|\sum_{1}^{p} h^{i}(x)\right\|},
$$

for every $x \in S^{n}$. Note that by hypothesis $\beta$ is well-defined and continuous.

Our next step is to estimate the degree of $\beta$. For that purpose, the following technical lemma will be useful.

Lemma 2.1 Let $X$ be a $n$-dimensional compact space, let $U \subset X$ be an open subset which is a topological $n$-manifold and let $f: X \rightarrow M$ be a continuous map into a $n$-dimensional manifold $M$. Suppose that there is $p \in M$ such that $f^{-1}(p) \subset U$. Then, we can approximate $f$ arbitrarily close by mappings $g: X \rightarrow M$ with the property that the restriction $g \mid: g^{-1}(V) \rightarrow V$ is a finite covering map, where $V \subset M$ is a neighborhood of $p$.

Proof We may assume, without loss of generality, that $f$ is a light map because every continuous map of a $n$-dimensional compact space into a $n$-dimensional manifold can by approximated by light mappings [2]. Since the preimage $f^{-1}(p)$ is a zero dimensional compact set, then it has neighborhoods whose connected components are very small. All these sufficiently small neighborhoods are embeddable in euclidean space and hence, we may assume that there is an open neighborhood $V$ of $f^{-1}(p)$ with a smooth structure. Then, we can approximate $f$ arbitrarily close by mappings $g: X \rightarrow M$ that coincide with $f$ outside $V$ and are smooth on $V$.

If $W=M-g(X-V)$, we have that for every $x \in W, f^{-1}(x) \subset V$. So, if $p_{0} \in W$ is a regular value of the restriction of $g$ to $V$, then $g^{-1}\left(p_{0}\right)$ is a finite set contained in $V$ and furthermore, there is a small neighborhood $\theta$ of $p_{0}$ in $W$ with the property that the restriction $g \mid: g^{-1}(\theta) \rightarrow \theta$ is a covering map. To conclude the proof, we consider the composition of $g$ with an automorphism of $M$ which sends $p_{0}$ to $p$. The composition has all promised properties and also can be chosen arbitrarily close to $f$.

Lemma 2.2 Let $h: S^{n} \rightarrow S^{n}$ be a periodic homeomorphism of prime period $p$, without balanced orbits and let $\beta: S^{n} \rightarrow S^{n}$ be its baricentric mapping. Then, the degree of $\beta$ is divisible by $p$.

Proof Let us denote by $S^{n} / h$ the orbit space of $h$ and by $\Pi: S^{n} \rightarrow S^{n} / h$ the natural projection. Because $p$ is prime, all nonfixed points of $h$ have orbits 
consisting of $p$ elements. Hence, the complement to the set of fixed points, in the orbit space, is a topological $n$-manifold.

By Lemma 2.1, we can approximate $\beta \Pi^{-1}: S^{n} / h \rightarrow S^{n}$ arbitrarily close by mappings $g: S^{n} / h \rightarrow S^{n}$ with the property that the restriction $g \mid: g^{-1}(V) \rightarrow$ $V$ is a finite covering map, where $V$ is an open subset of $S^{n}$ in the complement of the set of fixed points of $h$.

If $g$ is sufficiently close to $\beta \Pi^{-1}$, then $\beta$ is homotopic to $g \Pi$ and hence we can use $g \Pi$ and the fact that the restriction $g \Pi \mid: \Pi^{-1}\left(g^{-1}(V)\right) \rightarrow V$ is a finite covering map to calculate the degree of $\beta$. Note that the degree of $g \Pi$ is the sum of the signs of the preimages of a point in $V$, where the sign of a preimage is +1 if the orientation is locally preserved and -1 if the orientation is locally reversed. If $h$ is an orientation preserving homeomorphism, the corresponding sum for the finite covering map $\Pi \mid: \Pi^{-1}\left(g^{-1}(V)\right) \rightarrow g^{-1}(V)$ is $p$, because all elements of the same orbit have the same sign, therefore the whole sum for $g \Pi$ is a multiple of $p$. This implies that, in this case, the degree of $\beta$ is divisible by $p$.

If $p>2$, then $h$ is an orientation preserving homeomorphism and hence the degree of $\beta$ is divisible by $p$. If $p=2$ and $h$ is an orientation reversing homeomorphism, then $h$ has a balanced orbit, otherwise $h$ is homotopic to the identity. This concludes the proof of the lemma.

An application of Lemma 2.2 is the following well known fact [3].

Corollary 2.3 Let $h: S^{n} \rightarrow S^{n}$ be a periodic homeomorphism of period 2 . Then there exists an $x \in S^{n}$ such that $h(x)=-x$.

Proof Suppose that $h(x) \neq-x$, for all $x \in S^{n}$. Then, the baricentric mapping $\beta$ for $h$ can be defined and, by Lemma 2.2, has degree divisible by 2 . On the other hand $\beta$ is homotopic to the identity, because $\beta(x) \neq-x$, for all $x \in S^{n}$. This is a contradiction because, in this case, the degree of $\beta$ is 1 .

Lemma 2.4 Let $h: S^{n} \rightarrow S^{n}$ be a periodic homeomorphism of prime period $p$. Then there exists an $x \in S^{n}$ and $\lambda \geq 1$ such that:

$$
\lambda x+\sum_{1}^{p-1} h^{i}(x)=0 .
$$

Algebraic 83 Geometric Topology, Volume 1 (2001) 
Proof Suppose not. Hence, $\sum_{1}^{p} h^{i}(x) \neq 0$, for every $x \in S^{n}$ and therefore, by Lemma 2.2, the baricentric mapping $\beta$ of $h$ is well defined and has degree divisible by $p$. In particular, $\beta$ is not homotopic to the identity. Consequently, there exists an $x \in S^{n}$ with the property that $\beta(x)=-x$, that is, $\sum_{1}^{p} h^{i}(x)=$ $-\delta x$, for $\delta>0$, but hence, $(1+\delta) x+\sum_{1}^{p-1} h^{i}(x)=0$.

Corollary 2.5 Let $h: S^{n} \rightarrow S^{n}$ be a periodic homeomorphism. Then there exists an $x \in S^{n}$ such that the origin lies in the convex hull of $h^{*}(x)$.

The following geometric lemma will be used in the proof of Theorem 1.1.

Lemma 2.6 Let $\left\{x_{1}, \ldots, x_{p}\right\} \subset S^{n}$ be such that $\lambda x_{1}+\sum_{2}^{p} x_{i}=0$, for some $\lambda \geq 1$, and for $1 \leq i \leq p,\left\|x_{i+1}-x_{i}\right\| \leq \rho_{p}$, where $x_{p+1}=x_{1}$. Then $\left\{x_{1}, \ldots, x_{p}\right\}$ are the vertices of a planar regular convex $p$-gon.

Proof Let $\left\{q_{1}, \ldots, q_{p}\right\} \subset S^{1} \subset S^{n}$ be the ordered vertices of a regular convex $p$-gon. Suppose first that $p=2 k$. Assume without loss of generality that $x_{1}=$ $p_{1}=(1,0, \ldots, 0)$. Let $\pi: R^{n+1} \rightarrow R^{1}$ be the orthogonal projection. Since $\left\|x_{i+1}-x_{i}\right\| \leq \rho_{p}$, for every $i=1, \ldots, p$, and $x_{1}=p_{1}=(1,0, \ldots, 0)$, we have that $\pi\left(q_{p-1}\right) \leq \pi\left(x_{p-1}\right)$ and $\pi\left(q_{2}\right) \leq \pi\left(x_{2}\right)$. By the same reason, $\pi\left(q_{i}\right) \leq$ $\pi\left(x_{i}\right)$, for $i=1, \ldots, p$. Since, by hypothesis, $\sum_{1}^{p} \pi\left(x_{i}\right) \leq 0=\sum_{1}^{p} \pi\left(q_{i}\right)$, then $\pi\left(q_{i}\right)=\pi\left(x_{i}\right), i=1, \ldots, p$. Consequently $\left\|x_{i+1}-x_{i}\right\|=\rho_{p}$, for $i=1, \ldots, p$. Furthermore, we also have that $\left\{x_{1}, \ldots, x_{k+1}\right\}$ lies in a plane and similarly $\left\{x_{k+1}, \ldots, x_{2 k}\right\}$ also lies in a plane. Finally, in order to show that the set $\left\{x_{1}, \ldots, x_{p}\right\}$ is planar, note that its baricentre lies in $R^{1}$. The case $p=2 k+1$ is similar.

Next, we have the following Jung's Theorem [1] for spheres.

Lemma 2.7 Let $F \subset S^{n}$ be a set with diameter smaller than $t_{n}$. Then, $F$ is contained in a spherical $n$-disk of radius $\delta_{n}, \delta_{n}^{2}+t_{n}^{2}=4$. In particular, the convex hull of $F$ does not contain the origin.

Proof Suppose first that $F$ is a finite set with at most $(n+1)$ points. Then, exactly as in the euclidean version of Jung's Theorem [1], there is a spherical $n$-disk of radius $\delta_{n}$ containing $F$.

Let now $x_{0} \in F$ and suppose without loss of generality that $F$ is compact. Let $\Lambda$ be the spherical $n$-disk of radius $t_{n}$ centered at $x_{0}$ and let $\Delta$ be the complementary spherical $n$-disk of radius $\delta_{n}$ centered at $-x_{0}$. Clearly $F \subset$ 
int $\Lambda$. For every point $x \in F$, let $D_{x}$ be the spherical $n$-disk of radius $\delta_{n}$ centered at $x$. Note that $D_{x} \subset S^{n}-\left\{-x_{0}\right\}$, for every $x \in F$. By the first paragraph of this proof, for every subset $F^{\prime} \subset F$ with at most $(n+1)$ points, $\cap\left\{D_{x} \mid x \in F^{\prime}\right\} \neq \emptyset$. Using stereographic projection from $-x_{0}$ and Helly's Theorem, we conclude that $\cap\left\{D_{x} \mid x \in F\right\} \neq \emptyset$, and hence that there is a spherical $n$-disk of radius $\delta_{n}$ containing $F$.

\section{The proof of the theorems}

Theorem 1.1 follows immediately from Lemmas 2.4 and 2.6.

Proof of Theorem 1.2 The proof of the first part of the theorem follows from Corollary 2.5 and Lemma 2.7. Let $n \neq 1,3,7$, and let $h: S^{n} \rightarrow S^{n}$ be a periodic homeomorphism with the property that $\Theta(h)<t_{n-1}$.

Let $x \in S^{n}$ and let $\Gamma \subset h^{*}(x)$, be a set of at most $(n+1)$ points. Let $H$ be a hyperplane through the origin containing $\Gamma$ and suppose $S^{n-1}=H \cap S^{n}$. Since $\Theta(h)<t_{n-1}$, then the diameter of $\Gamma$ is smaller than $t_{n-1}$ and by Lemma 2.7, the origin is not in the convex hull of $\Gamma$.

On the other hand, by Corollary 2.5, there exists an $x_{0} \in S^{n}$ such that the origin lies in the convex hull of $h^{*}\left(x_{0}\right)$. If the period $p$ of $h$ is smaller than or equal to $n+1$, this is a contradiction. Suppose hence that $p \geq n+2$.

By Carathéodory's Theorem and the above, let $\Delta_{0}=\left\{h^{i_{0}}\left(x_{0}\right), \ldots, h^{i_{n+1}}\left(x_{0}\right)\right\} \subset$ $h^{*}\left(x_{0}\right)$ be a set of at most $(n+2)$ points with the property that the origin lies in the interior of the convex hull of $\Delta_{0}$. Hence, for every $x \in S^{n}$, the set $\Delta_{x}=\left\{h^{i_{0}}(x), \ldots, h^{i_{n+1}}(x)\right\} \subset h^{*}(x)$ has the property that the origin lies in the interior of the convex hull of $\Delta_{x}$, otherwise, by continuity, there should be a point $x^{\prime} \in S^{n}$ and $\Gamma^{\prime} \subset h^{*}\left(x^{\prime}\right)$, a set of at most $(n+1)$ points, with the property that the origin lies in the convex hull of $\Gamma^{\prime}$, which is a contradiction. By making $y=h^{i_{0}}(x)$, we have that there are $n+1$ integers $0<j_{1}<\ldots<j_{n+1}<p$, such that for every $y \in S^{n}$, the set $\left\{y, h^{j_{1}}(y), \ldots, h^{j_{n+1}}(y)\right\} \subset h^{*}(y)$ has the property that the origin lies in the interior of the convex hull of $\left\{y, h^{j_{1}}(y), \ldots, h^{j_{n+1}}(y)\right\}$.

Since the origin belongs to the interior of $\left\{y, h^{j_{1}}(y), \ldots, h^{j_{n+1}}(y)\right\}$, for every $y \in S^{n}$, the orthogonal projection of $\left\{h^{j_{1}}(y), \ldots, h^{j_{n}}(y)\right\}$ onto the hyperplane perpendicular to $y$, gives rise to a trivialization of the tangent space of $S^{n}$, which implies that $S^{n}$ is parallelizable. This is a contradiction because $n \neq$ $1,3,7$. 
Proof of Theorem 1.3 Let $h: S^{n} \rightarrow S^{n}$ be a periodic homeomorphism of period 3. By Lemma 2.4 there is a point $x$ of the sphere such that the convex hull of its orbit represents a triangle containing the origin. Therefore, all angles of the triangle are less than $\pi / 2$ and there is one of them which is greater or equal to $\pi / 3$. Then the distance between vertices corresponding to this angle is greater or equal to $\sqrt{3}=d_{3}$.

Proof of Theorem 1.4 If the period of $h$ is 2 or 3 , the proof follows from Corollary 2.3 and Theorem 1.3. If the period $p=2 k$, then $h^{k}$ is a homeomorphism of period 2 and therefore, by Corollary 2.3, there exists an $x \in S^{1}$ such that $h^{k}(x)=-x$, but then $\Theta(h)=2>d_{p}$. Consequently, we shall assume that $p=2 k+1, k \geq 2$. Finally, we may assume that for $0 \leq i \leq 2 k, h^{i}(x) \neq-x$, otherwise there is nothing to prove. For any natural number $r \geq 0$, define $x_{r}=h^{i}(x)$, if $r=i \bmod (n)$ and $0 \leq i \leq 2 k$. Note that $x_{1}, \ldots, x_{2 k+1}$ are the vertices of a convex polygon inscribed in $S^{1}$. Let us define $A(r)$ as the length of the circular arc between $x_{r}$ and $x_{r+1}$ that does not contain any other vertex of the polygon.

We start proving that for every $x \in S^{1}$, there exists $1 \leq i \leq 2 k+1$, such that

$$
\sum_{j=0}^{k-1} A(i+j) \geq 2 \pi \frac{k}{2 k+1} .
$$

For that purpose, let us consider the following sum:

$$
\sum_{i=1}^{2 k+1} \sum_{j=0}^{k-1} A(i+j) .
$$

In this sum, each arc is consider $k$ times, so this sum is equal to $k \sum_{j=1}^{2 k+1} A(j)=$ $2 \pi k$. Therefore, one of its $2 k+1$ summands is greater or equal than $2 \pi \frac{k}{2 k+1}$.

Next, we shall prove that for every $x \in S^{1}$,

$$
\sum_{j=0}^{k-1} A(j)<\pi .
$$

If we suppose that $\sum_{j=0}^{k-1} A(j)>\pi$, then $\sum_{j=k}^{2 k-1} A(j)<\pi$. So, for some $y \in S^{1}$, between $x$ and $h^{k}(x)$, one gets the equality $\sum_{j=0}^{k-1} A(j)=\pi$, for the sum that corresponds to the orbit $h^{*}(y)$, but this means that $h^{k}(x)=-x$, which is a contradiction. 
Therefore, for every $x \in S^{1}$, there exists $1 \leq i \leq 2 k+1$, such that:

$$
\pi>\sum_{j=0}^{k-1} A(i+j) \geq 2 \pi \frac{k}{2 k+1} .
$$

These two inequalities implies that for every $x \in S^{1},\left\|h^{i}(x)-h^{i+k}(x)\right\| \geq d_{2 k+1}$, because the diameter of a regular $(2 k+1)$ polygon inscribed in $S^{1}$ is equal to the length of the chord joining the endpoints of the circular arc of length $2 \pi \frac{k}{2 k+1}$.

Our conjecture is that the orbital diameter of a homeomorphism $h: S^{n} \rightarrow S^{n}$, of prime period $p$, is at least $d_{p}$. For example, the conjecture holds for isometries (linear homeomorphisms) on $S^{n}$ due to the following result.

Let $h: R^{n} \rightarrow R^{n}$ be an isometry of prime period $p \neq 2$. Then there are linear subspaces, $H_{o}, H_{1}, \ldots, H_{\lambda}$, with $\operatorname{dim} H_{i}=2, i=1, \ldots, \lambda$, such that $R^{n}=H_{o} \oplus \bigoplus_{1}^{\lambda} H_{i}$ and $h=i d \oplus \bigoplus_{1}^{\lambda} h_{i}$, where $h_{i}: H_{i} \rightarrow H_{i}$ is an isometry of period $p$, that is, $h_{i}$ is a rotation with angle $2 \pi k_{i} / p, i=1, \ldots, \lambda$.

We sketch here a proof of the this result. For every $i=1, \ldots, \frac{p-1}{2}$, let $r_{i}$ : $R_{i}^{2} \rightarrow R_{i}^{2}$ be the rotation with angle $2 \pi i / p$. Let us consider $R^{p-1}=\bigoplus_{1}^{\frac{p-1}{2}} R_{i}^{2}$ and let $r=\oplus r_{i}: R^{p-1} \rightarrow R^{p-1}$. Then, it is not difficult to see that the orbit of the point $a=(1,01,0, \ldots, 1,0) \in R^{p-1}$ is a regular $(p-1)$-simplex. That is, if $a^{i}=h^{i}(a)$, then, for $i \neq j,\left\|a^{i}-a^{j}\right\|=c t e$ and $\left\{a^{1}, \ldots, a^{p}\right\}$ is a regular $(p-1)$ simplex inscribed in $S^{p-2}$ with baricenter $\sum_{1}^{p} a^{i}=0$. Note that $\left\{a^{1}, \ldots, a^{p-1}\right\}$ is a basis of $R^{p-1}$. Moreover, every $x \in R^{p-1}$ can be uniquely expressed as $x=\sum_{1}^{p} x_{i} a^{i}$, with $\sum_{1}^{p} x_{i}=1$, and $r(x)=\sum_{1}^{p} x_{i} a^{i+1}$.

Suppose first that $h: R^{n} \rightarrow R^{n}$ is an isometry of prime period $p \neq 2$, with the property that there exists an $x \in R^{n}$ such that $\sum_{1}^{p} h^{i}(x)=0$ and the orbit $\left\{h(x), \ldots, h^{p}(x)\right\}$ generates $R^{n}$. We shall prove that $n=2 m$ and there are linear subspaces, $H_{o}, H_{1}, \ldots, H_{\lambda}$, with $\operatorname{dim} H_{i}=2, i=1, \ldots, m$, such that $R^{n}=\bigoplus_{1}^{n} H_{i}$ and $h=\bigoplus_{1}^{\lambda} h_{i}$, where $h_{i}: H_{i} \rightarrow H_{i}$ is an isometry of period $p$ different from id.

For that purpose, let $\varphi: R^{p-1} \rightarrow R^{n}$ by the epimorphism given by $\varphi\left(a^{i}\right)=$ $h^{i}(x)$, for $i=1, \ldots, p-1$. Since $\sum_{1}^{p} a^{i}=\sum_{1}^{p} h^{i}(x)=0$, then $\varphi\left(a^{p}\right)=h^{p}(x)$. Note that $h \varphi=\varphi r: R^{p-1} \rightarrow R^{n}$. Let now $H_{i}=\varphi\left(R_{i}^{2}\right)$. Remember that $R^{p-1}=\bigoplus_{1}^{\frac{p-1}{2}} R_{i}^{2}$ and $r=\oplus r_{i}: R^{p-1} \rightarrow R^{p-1}$. Hence, $h \varphi=\varphi r_{i}: R_{i}^{2} \rightarrow H_{i}$. Consequently, $h \mid: H_{i} \rightarrow H_{i}$ is an isometry of period $p$. Furthermore, since $h \varphi=\varphi r_{i}: R_{i}^{2} \rightarrow H_{i}, h \mid: H_{i} \rightarrow H_{i}$ is not the identity and by the same reason, 
since $p \neq 2, \operatorname{dim} H_{i} \neq 1$. Finally, just note that for $i \neq j$, either $H_{i} \cap H_{j}=\{0\}$ or $H_{i}=H_{j}$.

Next assume that $h: R^{n} \rightarrow R^{n}$ is an isometry of prime period $p \neq 2$, with the property that for every $x \in R^{n}, \sum_{1}^{p} h^{i}(x)=0$. We shall prove that $n=2 m$ and there are linear subspaces, $H_{o}, H_{1}, \ldots, H_{\lambda}$, with $\operatorname{dim} H_{i}=2, i=1, \ldots, m$, such that $R^{n}=\bigoplus_{1}^{n} H_{i}$ and $h=\bigoplus_{1}^{\lambda} h_{i}$, where $h_{i}: H_{i} \rightarrow H_{i}$ is an isometry of period $p$.

For that purpose, let $\left\{x_{1}, \ldots, x_{n}\right\}$ be a basis for $R^{n}$ and let $\Gamma_{i}$ be the linear subspace of $R^{n}$ generated by $h^{*}\left(x_{i}\right)$. By the above $\Gamma_{i}=\bigoplus_{j} H_{j, i}, h \mid=\bigoplus_{j} h_{j, i}$, where $\left.h\right|_{H_{j, i}}=h_{j, i}: H_{j, i} \rightarrow H_{j, i}$ is an isometry of period $p$ different from the identity. Therefore, either $H_{j, i} \cap H_{k, l}=\{0\}$ or $H_{j, i}=H_{k, l}$.

The result follows immediately by considering the following two linear subspaces: $\Delta=\left\{z \in R^{n} \mid h(z)=z\right\}$ and $\Lambda=\left\{z \in R^{n} \mid z=h(x)-x\right\}$, for which $\Delta \oplus \Lambda=R^{n}$.

For example, the isometries of $S^{3}$ of period five are basically the rotations around a plane, the product with the quaternionics and the homeomorphism produced by a cyclic permutation of a 4 -simplex.

Corollary 3.1 Let $h: S^{n} \rightarrow S^{n}$ be an isometry of prime period $p$ different from the identity. Then $\Theta(h)=d_{p}$.

Proof We know that $\Theta(h) \geq d_{p}$. We shall prove that for every $x \in S^{n}$, the diameter of $h^{*}(x)$ is at most $d_{p}$. Suppose $\left\|h^{i}(x)-x\right\|>d_{p}$, for some $1<$ $i<\frac{p-1}{2}$. Since $h$ is an isometry and $h^{p-i}\left(h^{i}(x)-x\right)=x-h^{p-i}(x)$, then $\left\|h^{i}(x)-x\right\|=\left\|h^{p-i}(x)-x\right\|$. Let $H$ be the plane containing $\left\{x, h^{i}(x), h^{p-i}\right\}$. Then the circle $H \cap S^{n}$ contains the isosceles triangle $\left\{x, h^{i}(x), h^{p-i}\right\}$ and has radius at most 1 . Therefore, $b=\left\|h^{i}(x)-h^{p-i}(x)\right\|=\left\|h^{p-2 i}(x)-x\right\|<\rho_{p}$, which is a contradiction to Lemma 2.6, because if $j=p-2 i$ and $x_{k}=h^{k j}(x)$, $k=1, \ldots, p$, then $\left\|x_{k}-x_{k+1}\right\|=b<\rho_{p}$.

Question Does there exist a periodic homeomorphism of $S^{3}$ of period 5 such that the convex hull of any orbit represents a nondegenerate 4 -simplex containing the origin in its interior?

Acknowledgement This work was partially supported by Russian Basic Research Foundation (project 99-01-00009) and CONACYT (projects 32728-E and 28058-E). 


\section{References}

[1] L Danzer, B Grünbaum and V Klee, Helly's theorem and its relatives, in Convexity, Proc. Symposia in Pure Math., Vol. 7, (Amer. Math. Soc., Providence, RI) (1963) 101-180

[2] W Hurewich, Über Abblindungen von endlichdimensionalen Rāumen auf Teilmengen Cartesischer Rāume, Sitrungsber. Preuss. Acad. Wiss. 34 (1933) 754765

[3] D Montgomery and L Zippin, Topological Transformation Groups, Interscience, NY, 1955.

[4] MH A Newman, A theorem on periodic transformations of spaces, Quart. J. Math. 2 (1931) 1-8

Instituto de Matemáticas

National University of Mexico

Circuito Exterior

Ciudad Universitaria

México 04510 D.F.

Email: luis@matem.unam.mx

Received: 26 March 2001 Revised: 29 June 2001 